\title{
Dysphagia in the aging cardiovascular patient
}

\author{
Nicolas J. Mouawad ${ }^{1,2}$, Guneet S. Ahluwalia ${ }^{1}$ \\ ${ }^{1}$ Bay Heart \& Vascular, McLaren Bay Region, Bay City, MI, USA; ${ }^{2}$ Department of Surgery, Michigan State University, College of Human Medicine, \\ East Lansing, MI, USA \\ Correspondence to: Nicolas J. Mouawad, MD, MPH, MBA, RPVI. McLaren Bay Region, Bay Heart \& Vascular, Michigan State University, 1900 \\ Columbus Avenue, 4th Floor, South Tower, Bay City, MI 48708, USA. Email: nmouawad@gmail.com.
}

\begin{abstract}
Dysphagia is not a normal process of aging. Dysphagia aortica refers to external compression of the distal esophagus from an enlarged, ectatic or aneurysmal thoracic aorta leading to mechanical obstruction of the food bolus. It is a rare cause of dysphagia seen in the older population and predisposing risk factors include female gender, short stature, and kyphosis. We selected the case of an octogenarian with a significant cardiovascular history presenting with dysphagia where the diagnosis of dysphagia aortica was delayed but ultimately identified. Although rare, we urge and remind a high index of suspicion for clinicians treating this patient population.
\end{abstract}

Keywords: Dysphagia aortica; aneurysm; dysphagia

Submitted May 29, 2017. Accepted for publication Sep 18, 2017.

doi: $10.21037 /$ jtd.2017.10.59

View this article at: http://dx.doi.org/10.21037/jtd.2017.10.59

\section{Introduction}

Dysphagia is defined as the sensation of difficulty or discomfort in swallowing, usually as a symptom of a disease. It is generally classified in response to either liquids or solids, and the precipitant can either be secondary to motor dysfunction (weak peristalsis or uncoordinated contractions) or mechanical obstruction (either intra-luminal or external compression). Dysphagia is more frequent in older individuals, occurring in approximately $25 \%$ of hospitalized patients and in $30-40 \%$ of nursing home residents (1).

Dysphagia aortica is a mechanical dysphagia of the esophagus due to external compression from an ectatic or aneurysmal aorta. The incidence is rare, however, it appears predisposed to a variety of risk factors including female gender, older age, short stature, and spinal abnormalities such as kyphosis $(2,3)$.

Herein we present the case of an elderly male with dysphagia aortica and remind those caring for an older vascular patient population the necessity for a high index of suspicion for this disease process to allow appropriate and timely intervention.

\section{Case presentation}

An 82-year-old Caucasian male with a significant cardiovascular history presented with chronic episodes of dysphagia to solids that had progressively worsened to liquids. He had associated unintentional weight loss over several months, malaise and lethargy. He endorsed regurgitation, halitosis, and intermittent nausea. His history included ischemic cardiomyopathy, chronic atrial fibrillation, hypertension, hyperlipidemia, chronic renal failure, previous cerebrovascular accident, and a previous open repair of an infrarenal abdominal aortic aneurysm. Admission blood analysis was consistent with malnutrition without leukocytosis. Physical examination identified a cachectic, feeble looking male with irregular heart sounds and vesicular breath sounds.

The patient had previously undergone several admissions with medical evaluation including esophagoduodenoscopy (EGD) with serial dilations for dysphagia-he reported no symptomatic relief with these interventions. A barium swallow demonstrated "bird-beaking" distally (Figure 1A); biopsies were negative. Chest $\mathrm{X}$-ray identified significant 

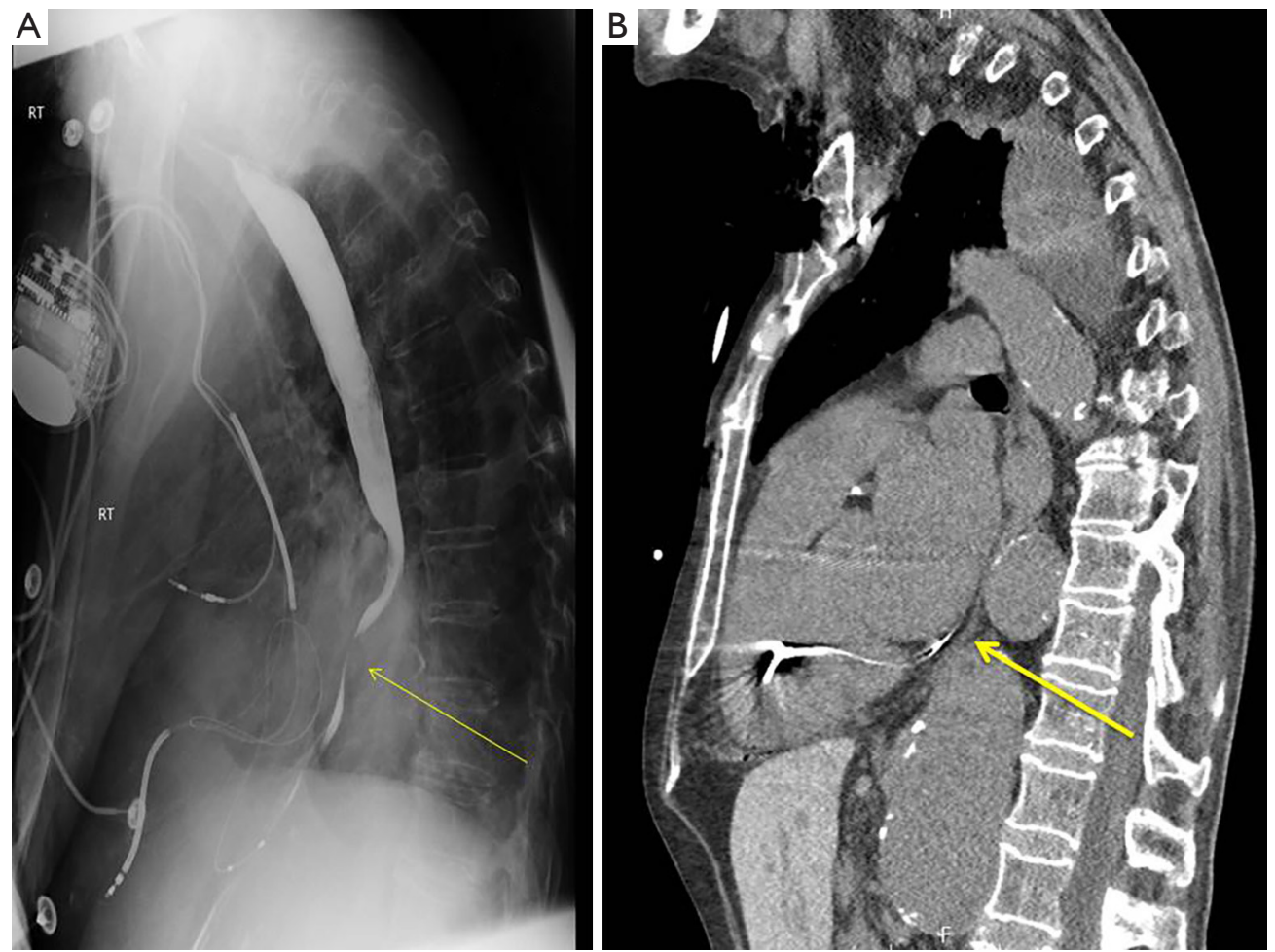

Figure 1 Barium esophagram demonstrating characteristic "bird-beaking" (A) and descending thoracic aneurysm component (B) with compression of the distal esophagus (as noted by yellow arrows).

spinal kyphosis with elements of congestive cardiac failure and fluid overload.

Vascular surgery was consulted due to the patient's multilevel vasculopathy - the diagnosis of dysphagia aortica was suggested at this point and a computed tomography scan identified a large $7.8 \mathrm{~cm}$ thoracoabdominal aortic aneurysm compressing the distal esophagus externally (Figures 1B,2). It was noted to be very tortuous and ectatic at the level of the diaphragmatic hiatus with a large paravisceral aortic component (Figure 2C). The patient was not a candidate for aortic repair given his significant malnutrition and comorbidities; a percutaneous endoscopic gastrostomy was placed and therapy was focused on medical optimization.

\section{Discussion}

Dysphagia aortica was first described by Pape in 1932 as external compression of the esophagus from an enlarged, ectatic, aneurysmal aorta (4). This aortic dilatation impinges on the anterolateral esophagus mechanically obstructing the bolus of food that should be propulsed distally. This is a pathologic condition as dysphagia is not a normal process of aging.

Risk factors that predispose to this disease entity include an older population, female sex, short stature, and kyphosis (3). Patients generally present with progressively worsening dysphagia, initially to solids and ultimately to liquids, including constitutional symptoms of nausea, anorexia, unintentional weight loss, and malnutrition. Laboratory analysis is unremarkable except for the catabolic state and hypoalbuminemia.

This diagnosis is usually completed by imaging modalities although no gold standard has been currently established. Chest radiographs can be helpful if the aorta is very calcified, suggesting aneurysmal degeneration with a severe atherosclerotic burden. Luminal evaluation with EGD may demonstrate pulsatile external compression within the distal esophagus and this can be supplanted with barium swallow where characteristic findings of esophageal wall indentation, partial esophageal obstruction, and pulsatile migration of the bolus chime is noted synchronous with aortic pulsation $(5,6)$. Clearly, these patients are elderly and may have multiple other confounding comorbidities, 


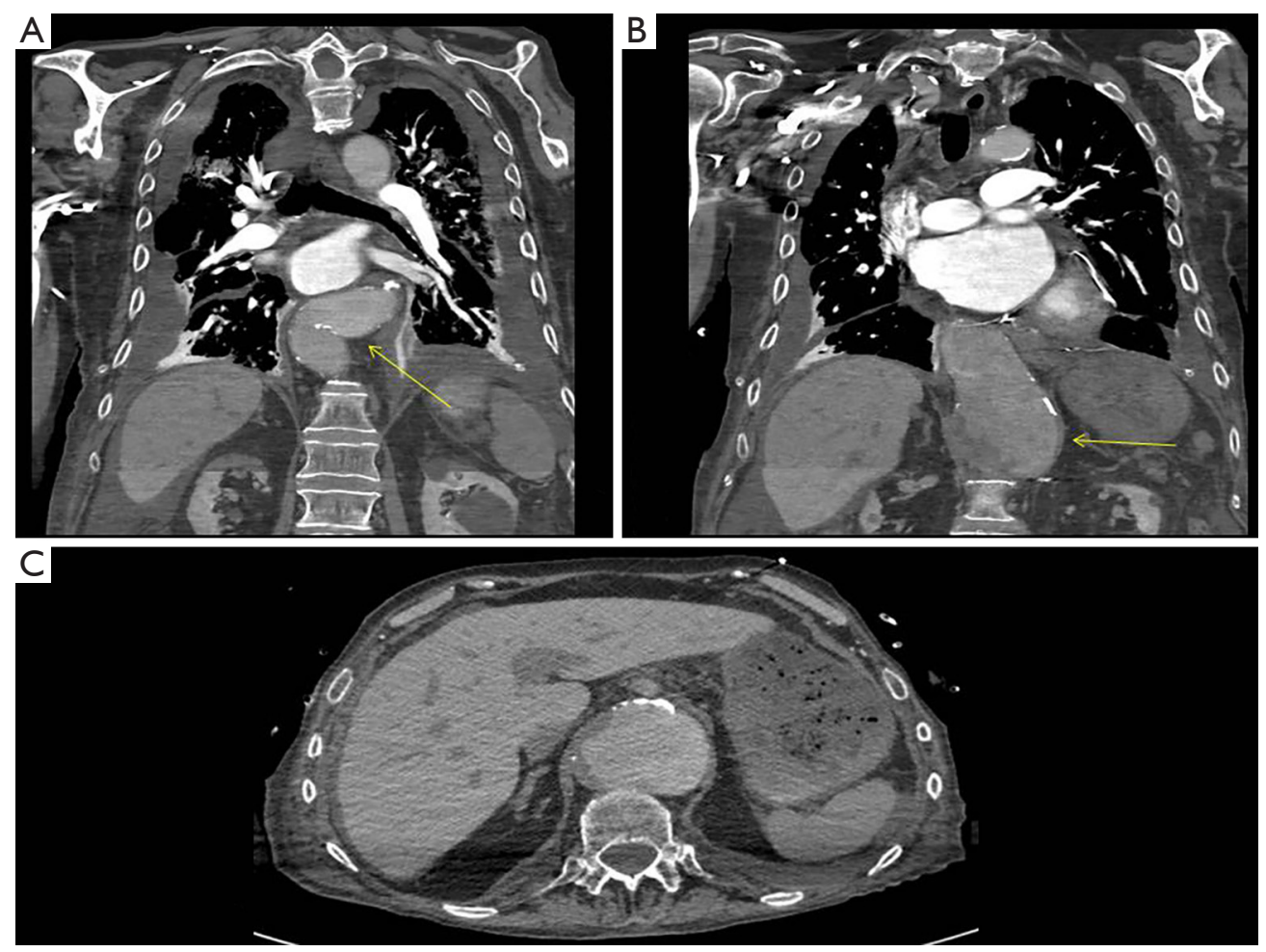

Figure 2 Contrast enhanced CT angiogram in coronal section of the distal thoracic aneurysm (A) and abdominal component (B) with severe tortuosity and kinking (yellow arrow); axial section of para-visceral extension (C).

therefore, any abnormality on barium evaluation should prompt an investigation for a neoplastic process if a filling defect is noted. Furthermore, some consider the use of esophageal manometry where a localized high-pressure band is also synchronous with the cardiac cycle-this finding, however, is nonspecific and inconsistent. Ultimately, however, computed tomography angiogram of the thorax is the most sensitive test to assist with diagnosis. It will identify the ectatic aorta and its compression onto the esophagus.

Treatment is aimed at symptom control. Unfortunately, the diagnosis is usually achieved too late and patients are too debilitated to undergo surgical correction with aneurysm repair, even via endovascular routes, as in our patient. Conservative measures are attempted first. Good nutritional support with diet supplements including diet modifications is important. Avoidance of large food boluses is helpful as well as posture mechanisms (avoidance of lying flat immediately after eating). The use of prokinetic agents has been demonstrated to be beneficial although the mechanism of action is unknown. Surgical correction may be performed via transposition of the distal esophagus, esophagomyotomy with division of the right crus of the diaphragm, or aortic resection and repair. Patients may even undergo PEG placement, esophageal dilations or even esophageal stenting for palliative measures $(7,8)$.

It is imperative that a high index of suspicion for dysphagia aortica is maintained in the older population presenting with dysphagia, in particular those with a cardiovascular history, as dysphagia is not a normal process of aging.

\section{Acknowledgements}

None.

\section{Footnote}

Conflicts of Interest: The authors have no conflicts of interest to declare.

Informed Consent: Written informed consent was obtained from the patient for publication of this case report and any accompanying images. 


\section{References}

1. Lindgren S, Janzon L. Prevalence of swallowing complaints and clinical findings among 50-79-yearold men and women in an urban population. Dysphagia 1991;6:187-92.

2. Wilkinson JM, Euinton HA, Smith LF, et al. Diagnostic dilemmas in dysphagia aortica. Eur J Cardiothorac Surg 1997;11;222-7.

3. Ponce J, Gaspar E, Del Val A, et al. Dysphagia of vascular origin. Rev Esp Enferm Dig 1991;80:53-6.

4. Pape R. Uber einen abnormen verlauf ('tiefe Rechtslage')

Cite this article as: Mouawad NJ, Ahluwalia GS. Dysphagia in the aging cardiovascular patient. J Thorac Dis 2017;9(11):E1005-E1008. doi: 10.21037/jtd.2017.10.59 der mesa aotitischen aorta descendens. Fortschr Roetgenstr 1932;46:257-69.

5. Coelho-Prabhu N, Baron TH. Dysphagia and weight loss in an elderly person. Dysphagia aortica. Gastroenterology 2009;137:e1-2.

6. Leonardi HK, Naggar CZ, Ellis FH Jr. Dysphagia due to aortic arch anomaly: diagnostic and therapeutic considerations. Arch Surg 1980;115:1229-32.

7. Song SW, Chung J, Kim SH. A case of dysphagia aortica in an elderly patient. Int J Gerontol 2012;6:46-8.

8. Hilliard AA, Murali NS, Keller AS. Dysphagia aortica. Ann Intern Med 2005;142:230-1. 